\title{
DISTORTION THEOREMS FOR BLOCH FUNCTIONS
}

\author{
XIANGYANG LIU AND DAVID MINDA \\ Dedicated to Professor Nobuyuki Suita on the occasion of his 60th birthday
}

\begin{abstract}
We establish various distortion theorems for both normalized locally schlicht Bloch functions and normalized Bloch function with branch points. These distortion theorems give lower bounds on either $\left|f^{\prime}(z)\right|$ or $\operatorname{Re} f^{\prime}(z)$; most of our distortion theorems are sharp and all extremal functions identified. The main tools used in establishing these distortion theorems are the classical form of Julia's Lemma and a new version of Julia's Lemma that applies to certain multiple-valued analytic functions. As applications of these distortion theorems, we obtain known lower bounds for various Bloch constants and also establish improved lower bounds on a number of Marden constants for Bloch, normal and Yosida functions.
\end{abstract}

\section{INTRODUCTION}

Assume that $f$ is a holomorphic function in the unit disk $\mathbb{D}$ of the complex plane. The function $f$ is called a Bloch function if and only if its Bloch seminorm given by $\|f\|=\sup \left\{\left(1-|z|^{2}\right)\left|f^{\prime}(z)\right|:|z|<1\right\}$ is finite.

For a point $a$ in $\mathbb{D}$, let $r(a, f)$ denote the radius of the largest schlicht disk on the Riemann surface $f(\mathbb{D})$ centered at $f(a)$ (a schlicht disk on $f(\mathbb{D})$ centered at $f(a)$ means that $f$ maps an open subset of $\mathbb{D}$ containing $a$ conformally onto this disk). Let $r(f)$ be the supremum of $r(a, f)$ for all $a$ in $\mathbb{D}$. The Bloch constant $B$ is defined to be the infimum of all $r(f)$, where $f$ is normalized by $f^{\prime}(0)=1$.

Bloch [4] proved that $B$ is positive. Landau [8] showed that in the definition of the Bloch constant, the infimum can be restricted to functions with Bloch seminorm 1. The exact value of the Bloch constant is still unknown. Ahlfors [2] and Heins [7] established the lower bound $B>\sqrt{3} / 4$. In 1988 Bonk [5] presented a simple proof of the following distortion theorem.

Received by the editors January 9, 1990 and January 16, 1990 and, in revised form, June 7, 1990. Presented at the Conference in Complex Analysis at Indiana University, September 17-18, 1989.

1980 Mathematics Subject Classification (1985 Revision). Primary 30C75; Secondary 30C25, 30C80, 30D45.

The research of the first author was partially supported by a Student Summer Research Fellowship awarded by the University Research Council of the University of Cincinnati, both authors' research partially supported by NSF Grant No. DMS-8801439. 
Bonk's Distortion Theorem. If $\|f\|=1$ and $f^{\prime}(0)=1$, then

$$
\operatorname{Re} f^{\prime}(z) \geq \frac{1-\sqrt{3}|z|}{(1-|z| / \sqrt{3})^{3}} \quad \text { for }|z| \leq \frac{1}{\sqrt{3}} \text {. }
$$

By making use of the above theorem, Bonk improved the lower bound on the Bloch constant to $B \geq \sqrt{3} / 4+10^{-14}$. Minda [13] gave an elementary, geometric proof of Bonk's result which yields all extremal functions. Actually, Bonk [6] had already determined all extremal functions, but by a different method as part of a larger study of Bloch functions.

Next, we review some facts about locally schlicht Bloch functions. A function $f$ is locally schlicht (or locally univalent) if and only if $f^{\prime}(z)$ does not vanish. The infimum of $r(f)$ for all locally schlicht functions normalized by $f^{\prime}(0)=1$ is called the locally schlicht Bloch constant and is denoted by $B_{\infty}$. Let $\mathscr{B}_{\infty}$ denote the family of locally schlicht functions satisfying $\|f\|=1, f(0)=0$ and $f^{\prime}(0)=1$. It is known that $B_{\infty}=\inf \left\{r(f): f \in \mathscr{B}_{\infty}\right\}$. The inequality $B_{\infty}>1 / 2$ was established by Ahlfors [2] and Pommerenke [17]. The best upper bound on the locally schlicht Bloch constant is (see [9])

$$
B_{\infty} \leq L \leq \frac{\Gamma\left(\frac{1}{3}\right) \Gamma\left(\frac{5}{6}\right)}{\Gamma\left(\frac{1}{6}\right)} \approx 0.5433,
$$

where $\Gamma$ denotes the Gamma function and $L$ is the so-called Landau constant. The number in the above inequality is conjectured to be the true value of $L$.

We use the geometric approach of Minda to establish an analog (Theorem 1) of Bonk's Distortion Theorem for locally schlicht Bloch functions in $\S 2$. As a corollary of our Theorem 1, we will give a new proof of the inequality $B_{\infty}>1 / 2$. The proof uses normal families and so is incapable of yielding an explicit lower bound for $B_{\infty}$ greater than $1 / 2$. In $\S 3$, we prove a generalization of Julia's Lemma for certain multiple-valued functions. Later in $\S 4$, Theorem 1 is extended to Bloch functions with branch points. Our Theorem 4 contains both Bonk's Distortion Theorem and Theorem 1 as special cases. Also, Theorem 4 yields the lower bounds for a number of the Bloch constants considered in [9]. Finally, in $\S 5$, we give improved lower bounds for various Marden constants for normal and Yosida functions as applications of our distortion theorems. These Marden constants were studied in [10] and [12].

\section{LOCALLY SCHLICHT BLOCH FUNCTIONS}

Versions of Julia's Lemma play an important role in our work. We begin by recalling a classical version of Julia's Lemma that is sufficient for our purposes, see [3, pp. 7-9] or [13] for details of its proof. For $r>0$, let

$$
\Delta(1, r)=\left\{z \in \mathbb{D}: \frac{|1-z|^{2}}{1-|z|^{2}}<r\right\}
$$

$\Delta(1, r)$ is a horodisk in $\mathbb{D}$, that is, a disk in $\mathbb{D}$ which is internally tangent to $\partial \mathbb{D}$ at 1 . In euclidean terms $\Delta(1, r)$ is the disk with center $1 /(1+r)$ and radius $r /(1+r)$. We let $\bar{\Delta}(1, r)$ denote the closure of $\Delta(1, r)$ relative to $\mathbb{D}$, so that $1 \notin \bar{\Delta}(1, r)$. If $T$ is a conformal automorphism of $\mathbb{D}$ which fixes 1 , then it is known that $T(\bar{\Delta}(1, r))=\bar{\Delta}(1, r)$. 
Julia's Lemma (disk version). Suppose that $w$ is a function holomorphic in $\mathbb{D} \cup\{1\}, w$ maps $\mathbb{D}$ into $\mathbb{D}$ and $w(1)=1$. Then $w^{\prime}(1)=c>0$ and for any $r>0$ the function $w$ maps the horodisk $\bar{\Delta}(1, r)$ into the horodisk $\bar{\Delta}(1, c r)$. Further, a point on the boundary of the first horodisk is mapped into the boundary of the second horodisk if and only if $w$ is a conformal automorphism of $\mathbb{D}$ which fixes 1 .

The following half-plane version of Julia's Lemma is more convenient for our purposes in this section. It follows from the disk version by composing the function with a Möbius transformation that maps the half-plane onto the unit disk and sends 0 to 1 .

Julia's Lemma (half-plane version). Suppose that $w$ is a function holomorphic in $\mathbb{D} \cup\{1\}, w$ maps $\mathbb{D}$ into the right half plane $\mathbb{H}=\{z: \operatorname{Re}(z)>0\}$ and $w(1)=0$. Then for any $r>0, w$ maps the horodisk $\bar{\Delta}(1, r)$ into the disk $\{z:|z-d r| \leq d r\}$, where $d=-w^{\prime}(1)>0$. Further, a point on the boundary of the first disk is mapped into the boundary of the second disk if and only if $w$ is a conformal mapping of $\mathbb{D}$ onto $\mathbb{H}$ which satisfies $w(1)=0$.

Corollary. For any $x \in(-1,1)$

$$
\operatorname{Re}(w(x)) \leq 2 d \frac{1-x}{1+x},
$$

with equality for some $x \in(-1,1)$ if and only if

$$
w(z)=2 d \frac{1-z}{1+z}
$$

Proof. Fix $x \in(-1,1)$ and let $r=(1-x) /(1+x)$. Then $r>0$ and $\bar{\Delta}(1, r)$ is the horodisk that contains the point $x$ on its boundary. The half-plane version of Julia's Lemma implies that $w(x)$ will lie inside the circle passing through the two points 0 and $2 d(1-x) /(1+x)$ and perpendicular to the real axis. The result follows.

Example. Now we consider a function that is an extremal function for Theorem 1. Let

$$
F(z)=-\frac{e}{2} \exp \left\{-\frac{1+z}{1-z}\right\}+\frac{1}{2} .
$$

Then $F$ is a holomorphic universal covering projection of $\mathbb{D}$ onto $\{w: 0<$ $|w-1 / 2|<e / 2\}$. Also,

$$
\left(1-|z|^{2}\right)\left|F^{\prime}(z)\right|=e \frac{\left(1-|z|^{2}\right)}{|1-z|^{2}} \exp \left\{-\frac{\left(1-|z|^{2}\right)}{|1-z|^{2}}\right\} \leq 1
$$

with equality if and only if $z$ lies on the circle $\left\{z:|1-z|^{2} /\left(1-|z|^{2}\right)=1\right\}=$ $\{z:|z-1 / 2|=1 / 2\}$, so $F$ has Bloch seminorm 1 .

Note that $F(0)=0$ and $F^{\prime}(0)=1$. Also, $r(0, F)=1 / 2$ because the open disk of radius $1 / 2$ centered at $F(0)=0$ is actually the largest schlicht disk in $F(\mathbb{D})$ centered at $F(0)$. Similarly, $r(F)=e / 4$ since $e / 4$ is the largest radius of a schlicht disk contained in $F(\mathbb{D})$. It is not difficult to verify that for all $z \in \mathbb{D}$,

$$
\left|F^{\prime}(z)\right| \geq F^{\prime}(|z|)=\frac{1}{(1-|z|)^{2}} \exp \left\{-\frac{2|z|}{1-|z|}\right\} .
$$


We also have

$$
\operatorname{Re} F^{\prime}(z)=\operatorname{Re}\left(\frac{1}{(1-z)^{2}} \exp \left\{-\frac{2 z}{1-z}\right\}\right)>0
$$

for $|z|<r$, where $r \approx 0.6958$ is the smallest root of the following equation.

$$
\frac{x \sqrt{\frac{1-x \sqrt{2-x^{2}}}{2}}}{1-x \sqrt{2-x^{2}}}-\arctan \left(\frac{x \sqrt{\frac{1-x \sqrt{2-x^{2}}}{2}}}{1-\frac{x^{2}+x \sqrt{2-x^{2}}}{2}}\right)=\frac{\pi}{4} \text {. }
$$

Theorem 1. Suppose $f \in \mathscr{B}_{\infty}$.

(a) For all $z \in \mathbb{D}$

$$
\left|f^{\prime}(z)\right| \geq F^{\prime}(|z|)=\frac{1}{(1-|z|)^{2}} \exp \left\{-\frac{2|z|}{1-|z|}\right\}
$$

with equality for some $z=r e^{i \theta} \neq 0$ if and only if $f(z)=e^{i \theta} F\left(e^{-i \theta} z\right)$ for some real $\theta$.

(b) For $|z| \leq 1 / 2$,

$$
\operatorname{Re} f^{\prime}(z) \geq F^{\prime}(|z|)=\frac{1}{(1-|z|)^{2}} \exp \left\{-\frac{2|z|}{1-|z|}\right\}
$$

with equality for some $z=r e^{i \theta} \neq 0$ if and only if $f(z)=e^{i \theta} F\left(e^{-i \theta} z\right)$ for some real $\theta$.

(c) Further, $\operatorname{Re} f^{\prime}(z)>0$ for $|z|<\sqrt{\pi /(4+\pi)} \approx 0.6633$.

Remark. The inequality in part (a), but not the identification of all extremal functions, was given by Peschl $[15,16]$. We will give a new proof of (a) which is simpler than Peschl's proof and also yields all extremal functions for this inequality. In addition, our technique of proof also yields the new results in parts (b) and (c).

Proof of Theorem 1. (a) Let

$$
g(z)=\left(\frac{1+z}{2}\right)^{2} f^{\prime}\left(e^{i \theta}\left(\frac{1-z}{2}\right)\right)
$$

where $\theta$ is real. Then $g$ is holomorphic in the closure of $\mathbb{D}$ except possibly at the point -1 . Further, $g(1)=1$ and $g \neq 0$. Since $f^{\prime}(0)=1$ and $\|f\|=1$, it follows that $f^{\prime \prime}(0)=0$. Hence, $g^{\prime}(1)=1$. We also have

$$
|g(z)|=\left|\frac{1+z}{2}\right|^{2}\left|f^{\prime}\left(e^{i \theta} \frac{1-z}{2}\right)\right| \leq\left(1-\left|\frac{1-z}{2}\right|^{2}\right)\left|f^{\prime}\left(e^{i \theta} \frac{1-z}{2}\right)\right| \leq 1
$$

for all $z$ in $\mathbb{D}$; here again we have used the fact that $\|f\|=1$. Note that the case $g=1$ is impossible since $g^{\prime}(1)=1 \neq 0$. So $g$ must map $\mathbb{D}$ into $\mathbb{D} \backslash\{0\}$.

Because $g$ never vanishes, there exists a holomorphic function $w$ which maps $\mathbb{D}$ into $\mathbb{H}$ such that $w(1)=0$, and $g(z)=\exp (-w(z))$. By direct calculation, $w^{\prime}(1)=-g^{\prime}(1)=-1$. From the corollary to the half-plane version of Julia's Lemma, we have

$$
|g(x)|=\exp \{-\operatorname{Re} w(x)\} \geq \exp \left\{-2 \frac{1-x}{1+x}\right\}
$$


for all $x$ in $(-1,1)$, with equality for some $x$ if and only if $w(z)=$ $2(1-z) /(1+z)$. Let $u=(1-x) / 2$, then the above inequality is equivalent to

$$
\left|f^{\prime}\left(e^{i \theta} u\right)\right| \geq \frac{1}{(1-u)^{2}} \exp \left\{-\frac{2 u}{1-u}\right\}
$$

for all $u \in(0,1)$, with equality for some $u$ if and only if $f(z)=e^{i \theta} F\left(e^{-i \theta} z\right)$ for some real $\theta$.

(b) As in the proof of (a), $w(x)$ will lie inside the circle $C$ with center $(1-x) /(1+x)$ and radius $(1-x) /(1+x)$. Since

$$
\min \{\operatorname{Re} \exp (-\zeta): \zeta \in C\}=\exp \left\{-2 \frac{1-x}{1+x}\right\}
$$

holds for all $x \in[0,1]$, we have

$$
\operatorname{Re} g(x)=\operatorname{Re} \exp \{-w(x)\} \geq \exp \left\{-2 \frac{1-x}{1+x}\right\}
$$

for all $x \in[0,1]$. This is equivalent to

$$
\operatorname{Re} f^{\prime}\left(e^{i \theta} u\right) \geq \frac{1}{(1-u)^{2}} \exp \left\{-\frac{2 u}{1-u}\right\}
$$

for all $u \in[0,1 / 2]$. The sharpness follows as in case (a).

(c) Set

$$
g(z)=\left(\frac{1-\alpha^{2}}{1-\alpha^{2} z}\right)^{2} f^{\prime}\left(e^{i \theta}\left(\frac{\alpha(1-z)}{1-\alpha^{2} z}\right)\right) \quad \text { with } \alpha=\sqrt{\frac{\pi}{4+\pi}} .
$$

As in the proof of part (a), $g$ is holomorphic in the closure of $\mathbb{D}$ and $g$ maps $\mathbb{D}$ into the punctured disk $\mathbb{D} \backslash\{0\}$. Further $g(1)=1$ and $g^{\prime}(1)=\pi / 2$. Hence there exists a holomorphic function $w$ which maps $\mathbb{D}$ into $\mathbb{H}$ such that $g(z)=\exp (-w(z)), w(1)=0$, and $w^{\prime}(1)=-\pi / 2$. The corollary to the halfplane version of Julia's Lemma shows that $w(x)$ will lie inside the circle $C$ centered at $\frac{\pi}{2}(1-x) /(1+x)$ with radius $\frac{\pi}{2}(1-x) /(1+x)$. Since

$$
\min \{\operatorname{Re} \exp (-\zeta): \zeta \in C\}>0
$$

holds for all $x \in(0,1], \operatorname{Re} g(x)>0$ for all $x \in(0,1]$. This is equivalent to $\operatorname{Re} f^{\prime}\left(e^{i \theta} u\right)>0$ for all $u \in[0, \sqrt{\pi /(4+\pi)})$.

Corollary. If $f$ satisfies the conditions of the above theorem, then $r(0, f) \geq 1 / 2$ with equality if and only if $f(z)=e^{i \theta} F\left(e^{-i \theta} z\right)$ for some real $\theta$. Further, $B_{\infty}>1 / 2$.

Proof. By definition, there is a simply connected open subset $\Omega$ of $\mathbb{D}$ containing 0 such that $f$ maps $\Omega$ univalently onto the disk centered at $f(0)$ with radius $r(0, f)$. This disk must touch the boundary of the Riemann surface $f(\mathbb{D})$. If not, then the boundary of $\Omega$ would be a Jordan curve inside $\mathbb{D}$. Because $f$ is locally schlicht, we could find an open subset of $\mathbb{D}$ containing the closure of $\Omega$ such that $f$ is schlicht on this larger set. Then the image of this larger set would contain a disk centered at $f(0)$ with radius larger than $r(0, f)$, which contradicts the definition of $r(0, f)$.

Hence, there is a radial segment $\Gamma$ in the above disk joining $f(0)$ to a boundary point of $f(\mathbb{D})$. Let $\gamma$ be the inverse image of $\Gamma$ under the mapping 
$f$, then $\gamma$ joins 0 to the boundary of $\mathbb{D}$. It follows from part (a) of Theorem 1 that

$$
r(0, f)=\int_{\Gamma}|d w|=\int_{\gamma}\left|f^{\prime}(z) d z\right| \geq \int_{0}^{1} F^{\prime}(|z|) d|z|=\frac{1}{2} .
$$

It is clear that equality holds in the above if and only if $f(z)=e^{i \theta} F\left(e^{-i \theta} z\right)$ for some real $\theta$.

Now we show that $B_{\infty}>1 / 2$. By a normal family argument, we can find an $f$ such that $\|f\|=1, f(0)=0, f^{\prime}(0)=1$, and $r(f)=B_{\infty}$. If $r(0, f)>1 / 2$, then $B_{\infty}=r(f) \geq r(0, f)>1 / 2$. If $r(0, f)=1 / 2$, then $f(z)=e^{i \theta} F\left(e^{-i \theta} z\right)$ for some real $\theta$, so $B_{\infty}=r(f)=r(F)=e / 4>1 / 2$.

\section{Generalization of Julia's Lemma}

In this section we obtain an extension of Julia's Lemma to certain multiplevalued functions.

We shall exploit the following connection between horodisks and hyperbolic disks. Let $D_{h}(a, r)=\left\{z: d_{h}(a, z)<r\right\}$ be the hyperbolic disk with center $a$ and hyperbolic radius $r$, where

$$
d_{h}(a, z)=2 \operatorname{artanh}\left|\frac{z-a}{1-\bar{a} z}\right|
$$

denotes the hyperbolic distance between $a$ and $z$ in $\mathbb{D}$. If $\left\{a_{n}\right\}$ is a sequence in $\mathbb{D}$ with $a_{n} \rightarrow 1,\left\{r_{n}\right\}$ is a sequence of positive numbers and

$$
\frac{1-\left|a_{n}\right|}{1-R_{n}} \rightarrow r, \quad \text { where } R_{n}=\tanh \left(\frac{r_{n}}{2}\right),
$$

then $D_{h}\left(a_{n}, r_{n}\right) \rightarrow \Delta(1, r)$. Note that $R_{n}$ is the pseudohyperbolic radius of $D_{h}\left(a_{n}, r_{n}\right)$. We refer to [3, pp. 7-8] for more details.

For $a \in \mathbb{D}$, define

$$
E_{a}(z)=\frac{1-\bar{a}}{1-a} \frac{z-a}{1-\bar{a} z} .
$$

Observe that $E_{a}$ is the only conformal automorphism of $\mathbb{D}$ which sends $a$ to 0 and fixes 1. Also $E_{a}^{\prime}(1)=\left(1-|a|^{2}\right) /\left(|1-a|^{2}\right)$. Therefore, $E_{a}^{\prime}(1) \leq c$ if and only if $a \notin \Delta(1,1 / c)$ and $E_{a}^{\prime}(1)=c$ if and only if $a$ belongs to $\partial \Delta(1,1 / c)$. Here $\partial \Delta(1,1 / c)$ denotes the boundary of $\Delta(1,1 / c)$ relative to $\mathbb{D}$, so $1 \notin \partial \Delta(1,1 / c)$.

Theorem 2. Suppose $g$ is holomorphic in $\mathbb{D} \cup\{1\}, g(\mathbb{D}) \subset \mathbb{D}$ and $g(1)=$ 1 . If all the zeros of $g$ have multiplicity at least $n$, then $g$ has no zero in $\Delta\left(1, n / g^{\prime}(1)\right)$ and $g$ has a zero on $\partial \Delta\left(1, n / g^{\prime}(1)\right)$ if and only if $g=E_{a}^{n}$ for some $a \in \partial \Delta\left(1, n / g^{\prime}(1)\right)$.

Proof. Assume $a \in \mathbb{D}$ and $g(a)=0$. Set $E=E_{a}$. Since $a$ is a zero of multiplicity at least $n, h=g / E^{n}$ is holomorphic on $\mathbb{D} \cup\{1\}$ and $h(1)=1$. There are two cases to consider. First, suppose $h$ is constant. Then $h=1$ and $g=E^{n}$. From $g^{\prime}(1)=n E^{\prime}(1)$ we see that $a \in \partial \Delta\left(1, n / g^{\prime}(1)\right)$. On the other hand, assume $h$ is nonconstant. Then $h^{\prime}(1)>0$ by Julia's Lemma, so $g^{\prime}(1)=h^{\prime}(1)+n E^{\prime}(1)>n E^{\prime}(1)$, or equivalently, $E^{\prime}(1)<g^{\prime}(1) / n$. Hence, $a \notin \bar{\Delta}\left(1, n / g^{\prime}(1)\right)$. 
Remark. The following observation will be used in the remainder of this section. If $g$ satisfies the hypotheses of Theorem 2, then $g^{1 / n}$ is a (possibly) multiplevalued holomorphic function on $\mathbb{D} \cup\{1\}$ with an algebraic branch point at any zero of $g$ which does not have multiplicity an integral multiple of $n$. However, since $g$ does not vanish in $\Delta\left(1, n / g^{\prime}(1)\right)$, there is a unique singlevalued holomorphic branch $G$ of $g^{1 / n}$ defined on $\Delta\left(1, n / g^{\prime}(1)\right) \cup\{1\}$ and normalized by $G(1)=1$. Note that $G^{\prime}(1)=g^{\prime}(1) / n$.

Theorem 3. Let $g$ be a function satisfying the hypotheses of Theorem 2 and let $G$ be the single-valued branch of $g^{1 / n}$ defined as above. Then for $0<r \leq n / g^{\prime}(1)$ the function $G$ maps $\bar{\Delta}(1, r)$ into $\bar{\Delta}\left(1, r G^{\prime}(1)\right)$. Moreover, a point of $\partial \Delta(1, r)$ is mapped into $\partial \Delta\left(1, r G^{\prime}(1)\right)$ if and only if $G$ is a conformal automorphism of $\mathbb{D}$ which fixes 1 .

Remark. Except for the restriction $r \leq n / g^{\prime}(1)$, the conclusion of Theorem 3 is the conclusion of Julia's Lemma if $G$ were holomorphic on all of $\mathbb{D}$.

Proof of Theorem 3. Because all zeros of $g$ have multiplicity at least $n$, the multiple-valued holomorphic function $g^{1 / n}$ satisfies the hypotheses of Nehari's generalization of Schwarz' Lemma (see [14] or [11]). In particular,

$$
\frac{\left|G^{\prime}(z)\right|}{1-|G(z)|^{2}} \leq \frac{1}{1-|z|^{2}}
$$

for all $z \in \Delta\left(1, n / g^{\prime}(1)\right)=\Delta$, and equality holds at a point if and only if $G$ is a conformal automorphism of $\mathbb{D}$.

Since $\Delta$ is hyperbolically convex and $G$ is single-valued, the above inequality implies that

$$
d_{h}(G(a), G(b)) \leq d_{h}(a, b)
$$

for all $a, b \in \bar{\Delta}$ with equality for $a \neq b$ if and only if $G$ is a conformal automorphism of $\mathbb{D}$. We may now conclude that $G\left(D_{h}(a, r)\right) \subset D_{h}(G(a), r)$ for any hyperbolic disk $D_{h}(a, r)$ which is contained in $\Delta$.

In order to complete the proof, we now follow a standard geometric proof of Julia's Lemma [3, pp. 8-9], being careful to work only in $\Delta$ where $G$ is singlevalued. Note that for $0<r \leq n / g^{\prime}(1)$, we have the inclusion $\bar{\Delta}(1, r) \subset \bar{\Delta}$. Let $x_{n}=1-1 / n$. Select $R_{n}$ so that $\left(1-x_{n}\right) /\left(1-R_{n}\right)=r$; that is, $R_{n}=1-1 /(n r)$. Set $r_{n}=2 \operatorname{artanh}\left(R_{n}\right)$. This guarantees that $D_{h}\left(x_{n}, r_{n}\right) \rightarrow \Delta(1, r)$. For all $n$ sufficiently large, $D_{h}\left(x_{n}, r_{n}\right) \subset \Delta$. For these large values of $n, G\left(D_{h}\left(x_{n}, r_{n}\right)\right) \subset$ $D_{h}\left(G\left(x_{n}\right), r_{n}\right)$. Because

$$
\frac{1-\left|G\left(x_{n}\right)\right|}{1-R_{n}}=\frac{1-x_{n}}{1-R_{n}} \frac{1-\left|G\left(x_{n}\right)\right|}{1-x_{n}} \rightarrow r G^{\prime}(1),
$$

we conclude that $D_{h}\left(G\left(x_{n}\right), r_{n}\right) \rightarrow \Delta\left(1, r G^{\prime}(1)\right)$. Consider $z \in \Delta(1, r)$. Then $z$ belongs to $D_{h}\left(x_{n}, r_{n}\right)$ for all $n$ sufficiently large. Hence, $G(z) \in$ $D_{h}\left(G\left(x_{n}\right), r_{n}\right)$ for all large $n$, so $G(z) \in \bar{\Delta}\left(1, r G^{\prime}(1)\right)$. This yields $G(\bar{\Delta}(1, r)) \subset$ $\bar{\Delta}\left(1, r G^{\prime}(1)\right)$.

Finally, we determine when a point on $\partial \Delta(1, r)$ can map into $\partial \Delta\left(1, r G^{\prime}(1)\right)$. Assume $a \in \partial \Delta(1, r)$ and $G(a) \in \partial \Delta\left(1, r G^{\prime}(1)\right)$. Let $\gamma$ be the hyperbolic geodesic through $a$ which ends at 1 . Select any $b \in \mathbb{D}$ on $\gamma$ strictly between $a$ and 1 . Determine $s \in(0, r)$ with $b \in \partial \Delta(1, s)$. Now, $d_{h}(a, b)$ is the hyperbolic distance between $\partial \Delta(1, r)$ and $\partial \Delta(1, s)$. Since $G(b)$ lies on 
or inside the circle $\partial \Delta\left(1, s G^{\prime}(1)\right), d_{h}(G(a), G(b))$ is greater than or equal to the hyperbolic distance from $\partial \Delta\left(1, r G^{\prime}(1)\right)$ to $\partial \Delta\left(1, s G^{\prime}(1)\right)$. But the hyperbolic distance between the two horocycles is the same as the hyperbolic distance between $\partial \Delta(1, r)$ and $\partial \Delta(1, s)$ since there is a conformal automorphism of $\mathbb{D}$ carrying the first pair of horocycles onto the second. Therefore, $d_{h}(G(a), G(b)) \geq d_{h}(a, b)$. Since the reverse inequality also holds, $G$ must be a conformal automorphism of $\mathbb{D}$ with $G(1)=1$.

Corollary. Suppose $g$ is holomorphic on $\mathbb{D} \cup\{1\}, g(\mathbb{D}) \subset \mathbb{D}, g(1)=1$ and all zeros of $g$ have multiplicity at least $n$.

(a) If $g^{\prime}(1)=n$, then $|g(x)| \geq x^{n}$ for $x \in[0,1)$ with equality for some $x$ if and only if $g(z)=z^{n}$.

(b) If $g^{\prime}(1)=n$, then $\operatorname{Re} g(x) \geq x^{n}$ for $(n-1) /(n+1) \leq x<1$ with equality for some $x$ if and only if $g(z)=z^{n}$.

(c) If $g^{\prime}(1)=n \sin (\pi / 2 n)$, then $\operatorname{Re} g(x)>0$ for $0<x<1$.

Remark. For $n=1$, part (b) implies both parts (a) and (c). For $n \geq 2$ this is no longer the case.

Proof. (a) Let $G$ be as in Theorem 3. Note that $G^{\prime}(1)=1$ and $G$ is defined on $\Delta(1,1)$, so $G(\bar{\Delta}(1, r)) \subset \bar{\Delta}(1, r)$ for $0<r \leq 1$. For $x \in[0,1), r=$ $(1-x) /(1+x) \in(0,1]$ and this containment yields $|G(x)| \geq \operatorname{Re} G(x) \geq x$, since $x \in \partial \Delta(1, r)$. Equality implies that $G$ is a conformal automorphism of $\mathbb{D}$ which fixes both $x$ and 1 , so $G$ is the identity mapping. Hence $|g(x)| \geq$ $|G(x)|^{n} \geq x^{n}$, with equality if and only if $g(z)=z^{n}$.

(b) As in part (a), we find that $\operatorname{Re} G(x) \geq x$ for $x \in[0,1)$ with equality if and only if $G(z)=z$. We wish to determine those $x \in[0,1)$ for which it follows that $\operatorname{Re} g(x) \geq x^{n}$.

The function $h(z)=1 /(1+r)+r z /(1+r)$ is a conformal mapping of $\mathbb{D}$ onto $\Delta(1, r)$. We determine when $h(z)^{n}=k(z)$ is a convex function. Since

$$
\operatorname{Re}\left\{1+z k^{\prime \prime}(z) / k^{\prime}(z)\right\}=\operatorname{Re}\left\{\frac{1+n r z}{1+r z}\right\}>\frac{1-n r}{1-r}, \quad|z|<1,
$$

is positive for $0<r \leq 1 / n$, the function $k(z)$ is convex for such $r$. Because the image of $k(z)$ is symmetric about the real axis, it will follow that

$$
\operatorname{Re} g(x)=\operatorname{Re} G(x)^{n} \geq \min \left\{\operatorname{Re}\left(w^{n}\right): w \in \bar{\Delta}(1, r)\right\}=k(-1)=x^{n},
$$

where $x=(1-r) /(1+r) \in[(n-1) /(n+1), 1)$.

(c) Now, the situation is slightly different from (b) since $G^{\prime}(1)=\sin [\pi /(2 n)]$ and $G$ is holomorphic in $\Delta(1,1 / \sin [\pi /(2 n)])$. For $r<1 / \sin [\pi /(2 n)]$ and $z \in \bar{\Delta}(1, r)$, we have $G(z) \in \bar{\Delta}(1, r \sin [\pi /(2 n)])$. For $w \in \bar{\Delta}(1, r \sin [\pi /(2 n)])$, we have the sharp inequality

$$
|\operatorname{Arg}(w)| \leq \sin ^{-1}(r \sin \pi / 2 n),
$$

so that

$$
\left|\operatorname{Arg}\left(w^{n}\right)\right| \leq n \sin ^{-1}(r \sin \pi / 2 n)<\pi / 2,
$$

if $0<r<1$. For $x \in(0,1), r=(1-x) /(1+x) \in(0,1)$ and so the preceding inequalities imply that

$$
|\operatorname{Arg}(g(x))|=\left|\operatorname{Arg}\left(G(x)^{n}\right)\right|<\pi / 2 .
$$




\section{BLOCH FUNCTIONS WITH BRANCH POINTS}

We establish a connection between Bonk's Theorem and Theorem 1. For any positive integer $n$, define

$$
\begin{array}{r}
\mathscr{B}(n)=\left\{f:\|f\|=1, f^{\prime}(0)=1, f(0)=0 \text { and if } f^{\prime}(a)=0 \text { for some } a \text { in } \mathbb{D},\right. \\
\text { then } \left.f^{(k)}(a)=0 \text { for } k=1,2, \ldots, n\right\} .
\end{array}
$$

That is, $\mathscr{B}(n)$ consists of those functions with Bloch norm 1 and all of whose branch points are of order at least $n+1$. Note that $\mathscr{B}(n) \supset \mathscr{B}(n+1)$, $\mathscr{B}(1)$ is the class of functions in Bonk's Distortion Theorem and $\mathscr{B}_{\infty}=$ $\bigcap\{\mathscr{B}(n): n=1,2,3, \ldots\}$.

Example. We now discuss functions $F_{n} \in \mathscr{B}(n)$ which have certain extremal properties. For $n \geq 1$, set

$$
F_{n}(z)=-\frac{n+2}{2(n+1)}\left(1+\frac{2}{n}\right)^{n / 2}\left(\frac{\sqrt{\frac{n}{n+2}}-z}{1-\sqrt{\frac{n}{n+2}} z}\right)^{n+1}+\frac{\sqrt{n(n+2)}}{2(n+1)} .
$$

Note that $F_{n}$ is holomorphic in $\mathbb{D}, F_{n}(0)=0, F_{n}^{\prime}(0)=1$ and

$$
F_{n}^{\prime}(z)=\left(1+\frac{2}{n}\right)^{n / 2} \frac{\left(\sqrt{\frac{n}{n+2}}-z\right)^{n}}{\left(1-\sqrt{\frac{n}{n+2}} z\right)^{n+2}} .
$$

We shall explicitly determine $\left\|F_{n}\right\|$ and $r\left(0, F_{n}\right)$.

In order to gain insight into the nature of the function $F_{n}$, it is useful to express $F_{n}$ in another form. Set

$$
T_{n}(z)=\frac{\sqrt{\frac{n}{n+2}}-z}{1-\sqrt{\frac{n}{n+2}} z} .
$$

Then $T_{n}$ is a conformal automorphism of $\mathbb{D}$ and

$$
F_{n}(z)=\frac{\sqrt{n(n+2)}}{2(n+1)}\left[1-\left(\frac{n+2}{n}\right)^{(n+1) / 2} T_{n}(z)^{n+1}\right] .
$$

So $F_{n}$ is an $(n+1)$-sheeted branched covering of $\mathbb{D}$ onto the disk with center

$$
F_{n}(\sqrt{n /(n+2)})=\sqrt{n(n+2)} /[2(n+1)]
$$

and radius

$$
\frac{n+2}{2(n+1)}\left(1+\frac{2}{n}\right)^{n / 2}
$$


Straightforward calculation gives

$$
\begin{aligned}
\left\|F_{n}\right\| & =\sup \left\{\left(1-|z|^{2}\right)\left|F_{n}^{\prime}(z)\right|:|z|<1\right\} \\
& =\left(1+\frac{2}{n}\right)^{n / 2} \sup \left\{\frac{\left(1-|z|^{2}\right)}{\left|1-\sqrt{\frac{n}{n+2}} z\right|^{2}}\left|T_{n}(z)\right|^{n}:|z|<1\right\} \\
& =\frac{n+2}{2}\left(1+\frac{2}{n}\right)^{n / 2} \sup \left\{\left(1-\left|T_{n}(z)\right|^{2}\right)\left|T_{n}(z)\right|^{n}:|z|<1\right\} \\
& =\frac{n+2}{2}\left(1+\frac{2}{n}\right)^{n / 2} \sup \left\{\left(1-|w|^{2}\right)|w|^{n}:|w|<1\right\}=1 .
\end{aligned}
$$

Next, we determine $r\left(0, F_{n}\right)$. Recall that $r\left(0, F_{n}\right)$ is the distance from $F_{n}(0)=0$ to the nearest branch point or to the boundary of $F_{n}(\mathbb{D})$, whichever is smaller. Since the only branch point, namely $F_{n}(\sqrt{n /(n+2)})$, is closer to 0 than 0 is to the boundary of $F_{n}(\mathbb{D})$, we have

$$
r\left(0, F_{n}\right)=\left|F_{n}(\sqrt{n /(n+2)})\right|=\frac{\sqrt{n(n+2)}}{2(n+1)} .
$$

Theorem 4. If $f \in \mathscr{B}(n)$, then

(a)

$$
\left|f^{\prime}(z)\right| \geq F_{n}^{\prime}(|z|) \quad \text { for }|z| \leq \sqrt{\frac{n}{n+2}}
$$

with equality for some $z=r e^{i \theta} \neq 0$ if and only if $f(z)=e^{i \theta} F_{n}\left(e^{-i \theta} z\right)$,

(b)

$$
\operatorname{Re} f^{\prime}(z) \geq F_{n}^{\prime}(|z|) \quad \text { for }|z| \leq \frac{n+2}{2 n+1} \sqrt{\frac{n}{n+2}}
$$

with equality for some $z=r e^{i \theta} \neq 0$ if and only if $f(z)=e^{i \theta} F_{n}\left(e^{-i \theta} z\right)$,

(c)

$$
\operatorname{Re} f^{\prime}(z)>0 \text { for }|z|<\sqrt{\frac{n \sin \frac{\pi}{2 n}}{2+n \sin \frac{\pi}{2 n}}} .
$$

Proof. (a) Let $g$ be defined as in the proof of Theorem 1(c) with $\alpha=\sqrt{n /(n+2)}$. That is,

$$
g(z)=\left(\frac{1-\frac{n}{n+2}}{1-\frac{n}{n+2} z}\right)^{2} f^{\prime}\left(e^{i \theta}\left(\frac{\sqrt{\frac{n}{n+2}}(1-z)}{1-\frac{n}{n+2} z}\right)\right),
$$

where $\theta$ is real. Then $g$ is holomorphic in the closure of $\mathbb{D}, g(\mathbb{D}) \subset \mathbb{D}$, $g(1)=1$, and $g^{\prime}(1)=n$. Also, all zeros of $g$ have multiplicity at least $n$. Corollary (a) of Theorem 3 gives

$$
|g(x)| \geq x^{n} \text { for all } x \in[0,1)
$$

with equality for some $x$ if and only if $g(z)=z^{n}$. This is equivalent to

$$
\left|f^{\prime}\left(e^{i \theta} u\right)\right| \geq F_{n}^{\prime}(u) \text { for } 0<u \leq \sqrt{\frac{n}{n+2}}
$$

with equality for some $u$ if and only if $f(z)=e^{i \theta} F_{n}\left(e^{-i \theta} z\right)$. 
(b) The proof is quite similar to that of part (a). Define $g$ as in part (a). Then Corollary (b) of Theorem 3 says that

$$
\operatorname{Re} g(x) \geq x^{n} \text { for } \frac{n-1}{n+1} \leq x<1
$$

with equality for some $x$ if and only if $g(z)=z^{n}$. This is equivalent to

$$
\operatorname{Re} f^{\prime}\left(e^{i \theta} u\right) \geq F_{n}^{\prime}(u) \quad \text { for } 0<u \leq \frac{n+2}{2 n+1} \sqrt{\frac{n}{n+2}}
$$

with equality for some $u$ if and only if $f(z)=e^{i \theta} F_{n}\left(e^{-i \theta} z\right)$.

(c) Define

$$
g(z)=\left(\frac{1-\alpha^{2}}{1-\alpha^{2} z}\right)^{2} f^{\prime}\left(e^{i \theta}\left(\frac{\alpha(1-z))}{1-\alpha^{2} z}\right)\right), \quad \alpha=\sqrt{\frac{n \sin \frac{\pi}{2 n}}{2+n \sin \frac{\pi}{2 n}}},
$$

where $\theta$ is real. We may apply Corollary (c) of Theorem 3 to obtain

$$
\operatorname{Re} g(x)>0 \text { for } 0<x<1 \text {. }
$$

This is equivalent to

$$
f^{\prime}\left(e^{i \theta} u\right)>0 \text { for } 0<u<\sqrt{\frac{n \sin \frac{\pi}{2 n}}{2+n \sin \frac{\pi}{2 n}}} .
$$

Remark. If we let $n \rightarrow \infty$ in Theorem 4, then we obtain Theorem 1, except we lose the sharpness by taking this limit. In particular, $F_{n} \rightarrow F$, where $F$ is the extremal function in Theorem 1. For the special case $n=1$, we obtain Bonk's Distortion Theorem and $F_{1}$ is the extremal function for Bonk's Theorem [13]. Also, if $B_{n}$ is the Bloch constant for the family $\mathscr{B}(n)$, then we can obtain the lower bound $B_{n}>\sqrt{n(n+2)} / 2(n+1)$ from Theorem 4 in the same manner as the corollary to Theorem 1 was proved. These lower bounds were originally obtained in [9] by a different method.

\section{LOWER BOUNDS FOR MARDEN CONSTANTS}

It is known that there exists a positive number $s$, such that any nonconstant Bloch function is schlicht on some hyperbolic disk in $\mathbb{D}$ with hyperbolic radius $s$. The Marden constant for Bloch functions, denoted by $M$, is the supremum of all such $s$. Similarly, we can define the Marden constant for locally schlicht Bloch functions and we denote it by $M_{\infty}$.

Minda gave lower bounds for $M$ and $M_{\infty}$ in [10]. Note that in [10] the Marden constant was defined in terms of pseudo-hyperbolic distance instead of hyperbolic distance. The lower bound $M \geq 2 \operatorname{artanh}(1 / \sqrt{3})$ is an immediate consequence of Bonk's Distortion Theorem (see [13]).

Although part (c) of Theorem 1 may not be best possible (as the extremal function $F$ possibly indicates), it still implies the following improvement of Minda's estimate on $M_{\infty}$ :

$$
M_{\infty} \geq 2 \operatorname{artanh} \sqrt{\pi /(4+\pi)}=1.5971 \ldots
$$

The proof of the above inequality is similar to the proof of Minda's lower bound, see [10] for details. 
Next, we consider normal functions. A meromorphic function $f$ in $\mathbb{D}$ is called a normal function if

$$
\alpha(f)=\sup \left\{\left(1-|z|^{2}\right)\left|f^{\prime}(z)\right| /\left(1+|f(z)|^{2}\right): z \in \mathbb{D}\right\}<\infty .
$$

It is easy to verify that the quantity $\alpha(f)$, called the order of the normal function $f$, is invariant under conformal automorphisms of $\mathbb{D}$ and rotations of the Riemann sphere (see the proof of Theorem 3 in [10]). If $f$ is a normal function and $a \in \mathbb{D}$, then set

$$
s(a, f)=\sup \left\{s: f \text { is schlicht in the hyperbolic disk } D_{h}(a, s)\right\}
$$

and

$$
s(f)=\sup \{s(a, f): a \in \mathbb{D}\} .
$$

The Marden constant for normal functions with order $\alpha(f)=m>0$ is given by

$$
M_{N}(m)=\inf \{s(f): f \text { is a normal function and } \alpha(f)=m\} .
$$

Similarly, the Marden constant for locally schlicht normal functions is given by $M_{N_{\infty}}(m)=\inf \{s(f): f$ is a locally schlicht normal function and $\alpha(f)=m\}$.

The following result improves the lower bounds on the Marden constants that were derived in [10].

Theorem 5. (a) $M_{N}(m) \geq 2 \operatorname{artanh}\left(1 / \sqrt{3\left(1+m^{2}\right)}\right)$.

(b) $M_{N_{\infty}}(m) \geq 2 \operatorname{artanh}\left(\sqrt{\pi /(4+\pi)\left(1+m^{2}\right)}\right)$.

Proof. We only give the proof of (a); the proof of (b) is the same except that Theorem 1(c) is needed instead of Bonk's Distortion Theorem. Let $f$ be a normal function with $\alpha(f)=m$. We want to show that

$$
s(f) \geq 2 \operatorname{artanh}\left(1 / \sqrt{3\left(1+m^{2}\right)}\right) .
$$

First we assume that $f(0)=0$ and $f^{\prime}(0)=m$. Then

$$
\arctan (|f(z)|) \leq \int_{0}^{z} \frac{\left|f^{\prime}(\zeta)\right|}{1+|f(\zeta)|^{2}}|d \zeta| \leq m \int_{0}^{z} \frac{|d \zeta|}{1-|\zeta|^{2}}=\frac{m}{2} \log \left(\frac{1+|z|}{1-|z|}\right),
$$

where the integrals are taken along the line segment from 0 to $z$. The preceding inequality shows that $f$ is holomorphic in the euclidean disk centered at 0 with radius $R=\tanh (\pi /(2 m))$. Let $r=1 / \sqrt{1+m^{2}}$, then $r<R$ and $F(z)=$ $f(r z) /(r m)$ is holomorphic in $\mathbb{D}$. We can verify that $F(0)=0$ and $F^{\prime}(0)=1$. By the above inequality, for any $z \in \mathbb{D}$,

$$
\begin{aligned}
\left(1-|z|^{2}\right)\left|F^{\prime}(z)\right| & =\frac{\left(1-|z|^{2}\right)}{m}\left|f^{\prime}(r z)\right| \leq \frac{\left(1-|z|^{2}\right)}{\left(1-|r z|^{2}\right)}\left(1+|f(r z)|^{2}\right) \\
& \leq \frac{\left(1-|z|^{2}\right)}{\left(1-|r z|^{2}\right)}\left(1+\tan ^{2}\left(\frac{m}{2} \log \frac{1+|r z|}{1-|r z|}\right)\right) \leq 1 .
\end{aligned}
$$

So $\|F\|=1$. By Bonk's Distortion Theorem, $F$ is schlicht in the disk $\{z$ : $|z|<1 / \sqrt{3}\}$. Hence $f$ is schlicht in the disk $\{z:|z|<r / \sqrt{3}\}$. This implies that

$$
s(f) \geq s(0, f) \geq 2 \operatorname{artanh}\left(\frac{1}{\sqrt{3\left(1+m^{2}\right)}}\right) .
$$


The proof for the general case is the similar to the proof of the corresponding result in [10].

A meromorphic function $f$ in the complex plane $\mathbb{C}$ is called a Yosida function if

$$
\beta(f)=\sup \left\{\left|f^{\prime}(z)\right| /\left(1+|f(z)|^{2}\right): z \in \mathbb{C}\right\}<\infty .
$$

For a Yosida function $f$, let

$s_{e}(f)=\sup \{s: f$ is schlicht in a euclidean disk in $\mathbb{C}$ with euclidean radius $s\}$.

The Marden constant for Yosida functions with $\beta(f)=m>0$ is defined by

$$
M_{Y}(m)=\inf \left\{s_{e}(f): f \text { is a Yosida function and } \beta(f)=m\right\} .
$$

It is known that $M_{Y}(m)=M_{Y}(1) / m$ [12]. The following theorem is a slight improvement of the corresponding result in [12].

Theorem 6. $M_{Y}(m) \geq 1 /(\sqrt{3} m)$.

Proof. We need only show that for any Yosida function $f$ with $\beta(f)=1$, it follows that $s_{e}(f) \geq 1 / \sqrt{3}$.

As in [12], we may assume $f(0)=0$ and $f^{\prime}(0)=1$. Since

$$
\arctan (|f(z)|) \leq \int_{0}^{z} \frac{\left|f^{\prime}(\zeta)\right|}{1+|f(\zeta)|^{2}}|d \zeta| \leq \int_{0}^{z}|d \zeta|=|z|,
$$

$f$ is holomorphic in $\mathbb{D}$; here again the integrals are taken along the line segment from 0 to $z$. The above inequality implies that

$$
\begin{aligned}
\left(1-|z|^{2}\right)\left|f^{\prime}(z)\right| & \leq\left(1-|z|^{2}\right)\left(1+|f(z)|^{2}\right) \\
& \leq\left(1-|z|^{2}\right)\left[1+\tan ^{2}(|z|)\right] \leq 1
\end{aligned}
$$

for all $z \in \mathbb{D}$. Hence $f$ is also a Bloch function with $\|f\|=1$. Bonk's Distortion Theorem shows that $f$ is schlicht in the disk $\{z:|z|<1 / \sqrt{3}\}$; this is what we want to prove.

\section{CONCLUding COMMENTS}

As in [13], one can give a geometric, Riemann surface interpretation for Theorems 1 and 4 and relate the extremal functions in these theorems to the ultrahyperbolic metrics constructed in [9] to estimate the Bloch constants $B_{n}$. We do not give the similar details here.

In Theorems 1 and 4 we apparently did not (except for the case $n=1$ ) find the largest disk centered at the origin in which $\operatorname{Re} f^{\prime}(z)>0$. What is the largest such radius? Are the functions $F$ and $F_{n}$ still extremal for this problem? Recall that $s(0, f)$ denotes the hyperbolic radius of the largest hyperbolic disk centered at origin in which $f$ is schlicht. Then

$$
s(0, F)=\frac{\pi}{1+\sqrt{1+\pi^{2}}}=0.7311 \ldots,
$$

and

$$
s\left(0, F_{n}\right)=\frac{\sqrt{n(n+2)} \sin \left(\frac{\pi}{n+1}\right)}{1+\sqrt{1+n(n+2) \sin ^{2}\left(\frac{\pi}{n+1}\right)}} .
$$


What are the sharp lower bounds on $s(0, f)$ for the function classes in Theorems 1 and 4 ? Are the functions $F$ and $F_{n}$ still extremal for this problem? For $n=1$ the function $F_{1}$ gives the sharp lower bound on $s(0, f)$ for the functions in Bonk's Distortion Theorem.

Added in proof. There is an error in the purported proof that $B_{\infty}>1 / 2$ which is given in [17]. For details concerning the error, consult A. Yamada, Bounded analytic functions and metrics of constant negative curvature on Riemann surfaces, Kodai Math. J. 11 (1988), 317-324.

\section{REFERENCES}

1. L. V. Ahlfors and H. Grunsky, Über die Blochsche Konstante, Math. Z. 42 (1937), 671-673.

2. L. V. Ahlfors, An extension of Schwarz's lemma, Trans. Amer. Math. Soc. 43 (1938), 359364.

3. __ Conformal invariants: Topics in geometric function theory, McGraw-Hill, New York, 1973.

4. A. Bloch, Les théorèmes de M. Valiron sur les functions entières et la théorie de l'uniformisation, Ann. Fac. Sci. Univ. Toulouse 17 (1925).

5. M. Bonk, On Bloch's constant, Proc. Amer. Math. Soc. 110 (1990), 889-894.

6. __ Extremal Probleme bei Bloch-Funktionen, Dissertation, Technischen Universität, Carolo-Wilhelmina zu Braunschweig, 1988.

7. M. Heins, On a class of conformal metrics, Nagoya Math. J. 21 (1962), 1-60.

8. E. Landau, Uber die Bloch'sche Konstante und zwei verwandte Weltkonstanten, Math. Z. 30 (1929), 608-634.

9. D. Minda, Bloch constants, J. Analyse Math. 41 (1982), 54-84.

10. $\ldots$, Marden constants for Bloch and normal functions, J. Analyse Math. 42 (1982/83), 117-127.

11. L_ Lower bounds for the hyperbolic metric in convex regions, Rocky Mountain. J. Math. 13 (1983), 61-69.

12. __ Yosida functions, Proc. Sympos. Complex Analysis, Xian, China, May 21-27, 1987, Lectures on Complex Analysis, World Scientific, 1988, pp. 197-213.

13. _ The Bloch and Marden constants, Computational Methods and Function Theory, edited by E. B. Saff, L. C. Salinas and R. S. Varga, Proceedings Valparaíso, 1989, Lecture Notes in Math., vol. 1435, Springer-Verlag, Berlin, 1990, pp. 131-142.

14. Z. Nehari, A generalization of Schwarz' Lemma, Duke Math. J. 14 (1947), 1035-1049.

15. E. Peschl, Über die Verwendung von Differentialinvarianten bei gewissen Funktionenfamilien und die Übertragung einer darauf gegründeten Methode auf partialle Differentialgleichungen vom elliptischen Typus, Ann. Acad. Sci. Fenn. A I Math. 336/6 (1963), 2-22.

16. __ Über unverzweigte konforme Abbildungen, Österreich Akad. Wiss. Math.-Natur. Kl. S.-B. II 185 (1976), no. 1-3, 55-78.

17. Ch. Pommerenke, On Bloch functions, J. London Math. Soc. (2) 2 (1970), 689-695.

Department of Mathematical Sciences, University of Cincinnati, Cincinnati, Ohio 45221-0025

E-mail address, David Minda: minda@ucbeh.bitnet 